\title{
Lymphoepithelioma-like Carcinoma of the Renal Pelvis: A Case Report and Review of the Literature
}

\author{
Hyein Ahn · Jongmin Sim · Hyunsung Kim · Kijong Yi · Hulin Han · Yumin Chung · Abdul Rehman • Seung Sam Paik \\ Department of Pathology, Hanyang University College of Medicine, Seoul, Korea
}

Lymphoepithelioma is an undifferentiated epithelial tumor primarily described in the nasopharynx and characterized by syncytial nests of malignant epithelial cells with a prominent reactive lymphoid infiltrate. ${ }^{1}$ A carcinoma that shows similar histological features but arises outside the nasopharynx is called lymphoepithelioma-like carcinoma (LELC). LELC has been described in a variety of organs including salivary glands, thymus, lungs, stomach, skin, uterine cervix, breast, prostate, and the urinary tract. ${ }^{2}$ The renal pelvis is an extremely rare site for this tumor. To the best of our knowledge, only eight cases have been reported in the English literature and one case has been reported in the Korean literature. ${ }^{1-8}$ We recently experienced a case of LELC arising in the right renal pelvis in a 65-year-old woman.

\section{CASE REPORT}

The patient presented with asymptomatic gross hematuria. Computed tomography scan showed a dense enhancing soft tissue mass in the right renal pelvis and upper ureter with direct involvement of the renal parenchyma and hilar fat tissue. These findings were suggestive of invasive urothelial carcinoma. The patient underwent a laparoscopic radical nephroureterectomy. The resected specimen was bisected and revealed a grayish white colored tumor mass arising within the renal pelvis and upper ureter (Fig. 1). The tumor was extending into the adjacent hilar fat tissue. The mass measured $5.3 \times 2.5 \mathrm{~cm}$ in size. The right

\section{Corresponding Author}

Seung Sam Paik, M.D.

Department of Pathology, Hanyang University College of Medicine, 222 Wangsimni-ro, Seongdong-gu, Seoul 133-791, Korea

Tel: +82-2-2290-8252, Fax: +82-2-2296-7502, E-mail: sspaik@hanyang.ac.kr

Received: May 7, 2014 Revised: June 2, 2014

Accepted: June 17, 2014 kidney showed severe hydronephrosis with striking parenchymal atrophy. Microscopic examination showed syncytial clusters and sheets of anaplastic tumor cells in a background of dense lymphoid stroma (Fig. 2A). The large polygonal tumor cells had amphophilic cytoplasm with ill-defined cell borders, giving the tumor a syncytial appearance. The stroma showed a prominent inflammatory infiltrate consisting of small lymphocytes and plasma cells surrounding the tumor cell nests and individual tumor cells. Interestingly, the luminal portion of the pelvis showed a component of moderately differentiated squamous cell carcinoma with exuberant keratinization (Fig. 2B) in approximately $40 \%$ of the tumor. The adjacent urothelium showed dysplastic change. The anaplastic epithelial cell nests or individual tumor cells of the LELC component were strongly positive for cytokeratin (Fig. 3A) and p53 (Fig. 3B), however, negative for $\mathrm{p} 63$. The squamous cell carcinoma component showed strong positivity for p63 (Fig. 3C). The stromal lymphoid cells showed positivity for $\mathrm{CD} 45, \mathrm{CD} 3$, and CD20. Neoplastic cells were negative for Epstein-Barr virus (EBV)-encoded RNA (EBER) by in situ hybridization (Fig. 3D). The pathological stage was T3N0M0 and the patient was treated with surgical resection alone without adjuvant radiotherapy or chemotherapy. There has been no evidence of recurrence or metastasis of the disease during 6 months of follow-up after surgery.

\section{DISCUSSION}

In this report, we describe an exceedingly rare case of renal pelvic LELC with a distinct component of squamous cell carcinoma. Most cases of LELC of the urinary tract have been found in the urinary bladder and rare isolated cases have been reported in the urethra, ureter, and renal pelvis. ${ }^{2}$ The renal pelvis is an 
extremely rare site for LELC. Since the first LELC case arising in the renal pelvis had been reported in 1998 by Fukunaga and Ushigome, $^{7}$ only eight cases of renal pelvic LELC have been described in the English-language literature to date. In Korea, there

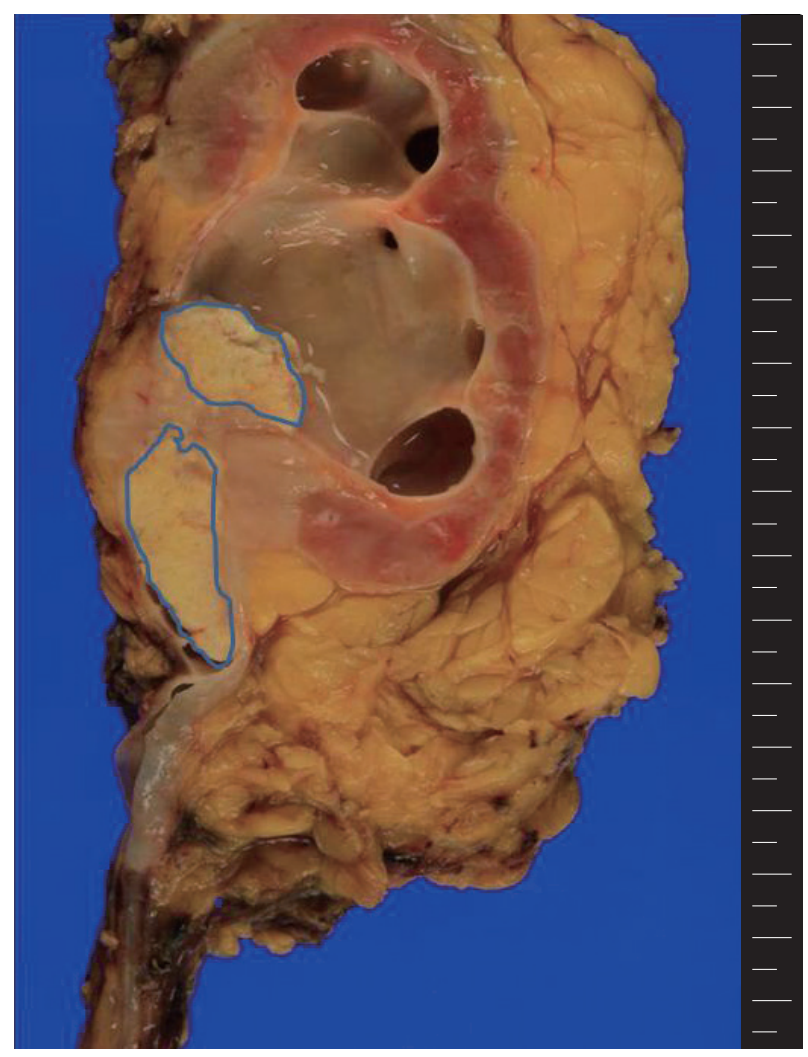

Fig. 1. A grayish white tumor is present in the renal pelvis with extension into the adjacent parenchyma and hilar fat tissue. The central whitish lesion reveals squamous cell carcinoma (blue color) and the peripheral solid portion reveal lymphoepithelioma-like carcinoma. has been one case of renal pelvic LELC described in the literature. The clinicopathological features of 10 LELC cases including our case are summarized in Table 1.

Recently, Modi et al. ${ }^{2}$ reported a case of LELC of the renal pelvis with aspects of pathological and therapeutic implications. They summarized the characteristics of the reported seven cases of LELC of the renal pelvis. The most common clinical presentation was frank hematuria. The age of patients ranged from 68 to 79 years with a mean age of 73.4 years. Of these seven patients, five were female and two were male. There were five patients manifested in advanced stage (T3) of disease with or without lymph node metastasis. All included cases expressed one or more markers including cytokeratin 7, cytokeratin 15, cytokeratin 20, and AE1/AE3, and none were associated with EBV infection. All patients received surgical treatment including the patient with adjuvant radiotherapy. The mean follow-up period was 20.1 months (range, 3 to 72 months). Follow-up results showed no evidence of disease in five patients and two patients died with disease.

According to the World Health Organization classification of urinary tract tumors, LELC has been classified as a distinct variant of urothelial carcinoma because it is derived from modified urothelial cells. LELC can be classified into three categories according to the proportion of LELC as pure (100\% LELC), predominant (more than 50\% LELC), and focal (less than 50\% LELC). ${ }^{2}$ Tamas et al. ${ }^{3}$ reported a clinicopathological study of 30 pure and mixed LELCs of the urinary tract. A total of 13 cases were predominant or focal LELCs with other patterns of carcinoma, including invasive urothelial carcinoma, invasive adenocarcinoma, and squamous cell carcinoma. In a review of report-
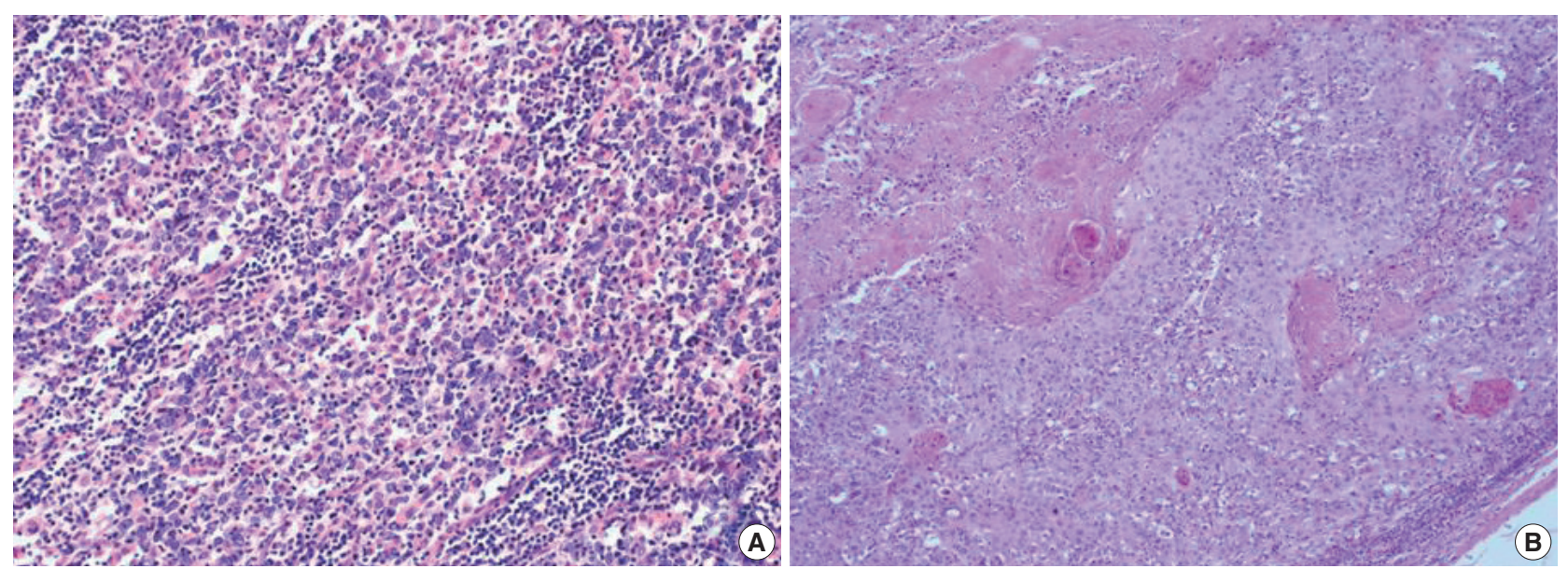

Fig. 2. The tumor shows syncytial clusters of large polygonal tumor cells with a background of dense lymphoid stroma (A) and squamous cell carcinoma component with exuberant keratin formation (B). 



Fig. 3. The tumor cells of lymphoepithelioma-like carcinoma are positive for cytokeratin (A) and p53 (B). The tumor cells of squamous cell carcinoma are positive for p63 (C). The tumor cells of lymphoepithelioma-like carcinoma are negative for Epstein-Barr virus-encoded RNA by in situ hybridization (D).

Table 1. The clinicopathological characteristics of reported lymphoepithelioma-like carcinoma of the renal pelvis

\begin{tabular}{|c|c|c|c|c|c|c|c|c|c|}
\hline Case No. & Sex/Age (yr) & Symptom & $\mathrm{HC}$ & Stage & EBV status & Tx & $F / U$ & Outcome & Reference \\
\hline 1 & $\mathrm{~F} / 75$ & Hematuria & Pure & T1N1M0 & Negative & $\mathrm{NU}$ & $36 \mathrm{mo}$ & NED & Haga et al. ${ }^{1}$ \\
\hline 2 & $\mathrm{~F} / 75$ & Hematuria & Predominant & T3N1M0 & Not tested & $\mathrm{NU}$ & $6 \mathrm{mo}$ & NED & Modi et al. ${ }^{2}$ \\
\hline 3 & Unknown & Unknown & Unknown & Unknown & Unknown & Unknown & Unknown & Unknown & Tamas et al. ${ }^{3}$ \\
\hline 4 & $\mathrm{~F} / 75$ & Hematuria & Unknown & T3NOMO & Negative & RN & $6 \mathrm{mo}$ & NED & Yamada et al. ${ }^{4}$ \\
\hline 5 & $\mathrm{~F} / 72$ & Unknown & Predominant & T3 & Negative & $\mathrm{RN}$ & $3 \mathrm{mo}$ & DWD & Perez-Montiel et al. ${ }^{5}$ \\
\hline 6 & $M / 68$ & Unknown & Focal & T3 & Negative & RN & $12 \mathrm{mo}$ & DWD & Perez-Montiel et al..$^{5}$ \\
\hline 7 & $\mathrm{~F} / 79$ & Hematuria & Unknown & T3NOMO & Negative & $\mathrm{NU}$ & $6 \mathrm{mo}$ & NED & Cohen et al. ${ }^{6}$ \\
\hline 8 & $\mathrm{M} / 70$ & Hematuria & Pure & Unknown & Negative & $\mathrm{RN}+\mathrm{RT}$ & $6 \mathrm{yr}$ & NED & Fukunaga and Ushigome ${ }^{7}$ \\
\hline 9 & $\mathrm{~F} / 70$ & Hematuria & Unknown & T2NOMO & Negative & NU & $24 \mathrm{mo}$ & NED & Hahm et al. ${ }^{8}$ \\
\hline 10 & $F / 65$ & Hematuria & Predominant & T3NOMO & Negative & $\mathrm{RN}$ & $6 \mathrm{mo}$ & NED & Present case \\
\hline
\end{tabular}

HC, histological classification; EBV, Epstein-Barr virus; Tx, treatment; F/U, follow-up; F, female; NU, nephroureterectomy; NED, no evidence of disease; RN, radical nephrectomy; DWD, died with disease; $\mathrm{M}$, male; RT, radiation therapy.

ed LELCs arising in the renal pelvis, most cases were pure LELCs, and there was a case with a mixed pattern of LELC and invasive urothelial carcinoma. However, there were no cases of the mixed pattern of LELC, adenocarcinoma, squamous cell carci- noma. Our case was a predominant type LELC with a combined histologic pattern of squamous cell carcinoma.

Almost all nasopharyngeal lymphoepitheliomas are tightly associated with EBV infection. Previous studies have evaluated 
the association between EBV and LELC of the urinary tract. ${ }^{9}$ Unlike nasopharyngeal lymphoepithelioma, EBV has not been identified by either in situ hybridization or immunohistochemistry in LELC of the urinary tract in previous studies. Haga et al. ${ }^{1}$ found that EBV-positive LELC tends to occur in organs that have a direct exposure to the external environment and EBVnegative tumors occur more often in internal organs. These data suggest an alternative etiology for LELC of urinary tract. Tamas et al. ${ }^{3}$ suggested that abnormalities of $\mathrm{p} 53$ regulation might be crucial in the development of LELC of the urinary tract. In our case, tumor cells expressed strong and diffuse positivity for nuclear p53 but were negative for EBER by in situ hybridization. Our result suggests a possible crucial role of $\mathrm{p} 53$ abnormality in the development of LELC of the urinary tract.

Although the existing data show that LELC most often manifest in stage T2 or T3, these tumors appeawr to offer greater patient survival than other types of urinary tract cancers. Previous smaller studies demonstrate that the pure or predominant subtype of LELC has a relatively favorable prognosis compared to focal LELC. ${ }^{10}$ Primary squamous cell carcinomas have been reported in the renal pelvis. Keratinizing squamous cell carcinoma makes up most cases and shows a high nuclear grade with an unfavorable prognosis. ${ }^{5}$ In our case, the tumor showed two distinct histologic patterns consisting of LELC and squamous cell carcinoma. The LELC was the dominant tumor component and showed infiltrative tumor growth into the renal parenchyma and adjacent hilar fat tissue. In the center of the tumor, the squamous cell carcinoma component was found with ureteropelvic obstruction resulting in hydronephrosis. Regardless of the isolated behavior of the LELC, other more aggressive squamous cell carcinoma components may play a critical role in prognosis. Our patient did not receive chemotherapy or radiotherapy after surgical treatment. Follow-up imaging studies showed no signs of recurrence or metastasis.

In summary, this is the first case report of mixed LELC of the renal pelvis with a distinct squamous cell carcinoma component. The recognition of this type of tumor is important for diagnostic implications to avoid misdiagnosis as a reactive inflammatory lesion or malignant lymphoma. The other type of carcinoma in mixed LELC may affect long-term prognosis. Further long-term follow-up is needed for to make judgments regarding prognosis to evaluate the exact character of this entity.

\section{Conflicts of Interest}

No potential conflict of interest relevant to this article was reported.

\section{REFERENCES}

1. Haga K, Aoyagi T, Kashiwagi A, Yamashiro K, Nagamori S. Lymphoepithelioma-like carcinoma of the renal pelvis. Int J Urol 2007; 14: 851-3.

2. Modi H, Beckley I, Bhattarai S, Spencer J, Cartledge J. Lymphoepithelioma-like carcinoma of the renal pelvis: pathological and therapeutic implications. Can Urol Assoc J 2013; 7: E590-3.

3. Tamas EF, Nielsen ME, Schoenberg MP, Epstein JI. Lymphoepithelioma-like carcinoma of the urinary tract: a clinicopathological study of 30 pure and mixed cases. Mod Pathol 2007; 20: 828-34.

4. Yamada Y, Fujimura T, Yamaguchi T, et al. Lymphoepithelioma-like carcinoma of the renal pelvis. Int J Urol 2007; 14: 1093-4.

5. Perez-Montiel D, Wakely PE, Hes O, Michal M, Suster S. High-grade urothelial carcinoma of the renal pelvis: clinicopathologic study of 108 cases with emphasis on unusual morphologic variants. Mod Pathol 2006; 19: 494-503.

6. Cohen RJ, Stanley JC, Dawkins HJ. Lymphoepithelioma-like carcinoma of the renal pelvis. Pathology 1999; 31: 434-5.

7. Fukunaga M, Ushigome S. Lymphoepithelioma-like carcinoma of the renal pelvis: a case report with immunohistochemical analysis and in situ hybridization for the Epstein-Barr viral genome. Mod Pathol 1998; 11: 1252-6.

8. Hahm YI, Yim SJ, Sim YJ, et al. Lymphoepithelioma-like carcinoma of the renal pelvis. Korean J Urol 2008; 49: 461-3.

9. Gulley ML, Amin MB, Nicholls JM, et al. Epstein-Barr virus is detected in undifferentiated nasopharyngeal carcinoma but not in lymphoepithelioma-like carcinoma of the urinary bladder. Hum Pathol 1995; 26: 1207-14.

10. Lopez-Beltran A, Luque RJ, Vicioso L, et al. Lymphoepitheliomalike carcinoma of the urinary bladder: a clinicopathologic study of 13 cases. Virchows Arch 2001; 438: 552-7. 